\title{
Am I better in VR with a real audience?
}

\author{
Romain Terrier ${ }^{1,2}$, Nicolas Martin ${ }^{1}$, Jeremy Lacoche ${ }^{1,3}$, Valerie Gouranton ${ }^{2}$, \\ and Bruno Arnaldi ${ }^{2}$ \\ 1 IRT $\mathrm{b}<>$ com, Rennes, France \\ firstname. lastname@b-com.com \\ 2 Univ Rennes, INSA Rennes, Inria, CNRS, IRISA, Rennes, France \\ 3 Orange Labs, Rennes, France
}

\begin{abstract}
We present an experimental study using virtual reality (VR) to investigate the effects of a real audience on social inhibition. The study compares a multi-user application, locally or remotely shared. The application engages one user and a real audience (i.e., local or remote conditions) and a control condition where the user is alone (i.e., alone condition). The differences have been explored by analyzing the objective performance (i.e., type and answering time) of users when performing a categorization of numbers task in VR. Moreover, the subjective feelings and perceptions (i.e., perceptions of others, stress, cognitive workload, presence) of each user have been compared in relation to the location of the real audience. The results showed that in the presence of a real audience (in the local and remote conditions), user performance is affected by social inhibitions. Furthermore, users are even more influenced when the audience does not share the same room, despite others are less perceived.
\end{abstract}

Keywords: Virtual Reality $\cdot$ Social influence $\cdot$ Audience

\section{Introduction}

The new trend is toward multi-users in Virtual Reality (VR) [30]. People are able to share, in real time, the same Virtual Environment (VE) and their experience causing social mechanisms [1] (e.g., social anxiety, social inhibition, empathy, group effects, leadership). Moreover, social VR applications allow people to share the same VE in different ways. On the one hand, they can be colocated when each user is in the same location. On the other hand, when each user is in a different, remote location, they are distant. This differentiation increases the possible social inhibition; indeed, the effects and the appearance of social inhibition are not well known in VR.Therefore, one question remains: does our behaviour change in VR depending on whether our audience is real or remote? Using previous experimental studies in psychology, we designed an experiment in which one user must perform a new and unknown task in front of distant or colocated others. In each condition, others are sharing the same VE as the user. The goal of this study is to analyze the differences in social inhibition depending on the location of the audience. 


\section{Related Work}

\subsection{Social influence and audience effects in the real world}

In the real world, everyday tasks are often done in presence of other people or with them. The perception one has of the other people affects his/her actions and behaviours [8] by imitation effects [5] or by a priming effect [25]. Firstly, the mere presence of other people (audience) is enough to influence individual behaviours [34] and to cause social facilitation [7]. The audience acts like an amplifier: it increases the dominant response - task complexity dependent - of the user. Thereby, the realization of an easy or well-known task will be facilitated by the mere presence of an audience, a concept known as facilitation [11]. In contrast, the realization of a complex or new task requiring a learning phase will be impaired [33], this is known as social inhibition. However, social inhibition or facilitation are not only generated by the mere presence of an audience [12]. How the audience is perceived or interpreted also affects the user, whose performance varies depending on the audience's status (i.e., evaluative or non-evaluative audience) [14]. Indeed, the user performs worse when facing an evaluative audience [16] than when alone because of the "evaluation apprehension" effect. A user must perceived an experimenter as an expert, and so evaluative, even if the experimenter is just an observer [27]. Also, knowing the identity of the audience (i.e., a friend) reduces accuracy and answering time given by users [32]. Social norm effects can also influence other behaviors: in a risky situation and in the presence of an audience users tend to diminish their risk-taking actions (e.g., poker gambling) [20]. Thus, many studies have been published on the social effects of an audience on a user (i.e., the mere presence, the evaluation apprehension, social norms, distraction, perception of the audience, predictability of the audience) and all refers to social facilitation or inhibition. Moreover, the social influence of a virtual audience on a user could be different when compared to the influence of a real audience due to the particularities of VR.

\subsection{Social inhibition in VR}

Researchers have used Shared Virtual Environments (SVEs)-inducing co-presence (i.e., "being there together" [22]) - to study users' behaviour in VR (e.g., paranoia, phobia, stress, anxiety) [21]. Participants are either physically in the same room (i.e., colocated) or in a remote place (i.e., distant). Several studies $[9,31]$ on social influence focus their scope on analysing social inhibition in VR. Social inhibition occurs when one performs a new or unknown task in front of an audience, resulting in a decrease in performance (e.g., less qualitative, less quantitative, slower) [3]. Many VR studies specialize their research in the type of the audience (e.g., real versus virtual in $2 \mathrm{D}$ or $3 \mathrm{D}$, close versus far). The first studies of the Social Inhibition of Return (e.g., increase in answering time in front of a co-actor) found that an agent in the VE caused a longer response time (e.g., 20ms) [31]. In a previous study, only the presence of a real co-actor caused this effect compared to a 2D-agent displayed on TV [23]. Moreover, a 
user study [15] focused on the difference of impact between three types of audiences: alone, with avatars, and with agents. The study is based on previous works of Blascovich et al. [2] who found that users had fewer correct answers when executing the unlearned task in presence of an audience. In the Hoyt et al. study [15], social inhibition occurred in VR but not social facilitation, and only in the presence of avatars. Indeed, if agents are judged and perceived as non-evaluative, the emergence of the inhibition can be prevented [7]. Moreover, agents exert less social influence than avatars on user feelings or behaviours [28] resulting in non-existant or low social inhibition. In the Hoyt et al. study [15], lower performance (e.g., lower number of correct answers during a novel task) was caused by social inhibition. However, the assistant stayed in the room even if the condition was without an audience, and that could have had an impact on results. There is still uncertainty regarding the effect of the audience type on users. Some results showed social inhibition in the presence of an agent while others showed social inhibition only in the presence of an avatar in a VE. One limitation of these previous studies is that the co-presence is not often measured, reducing data on the perception of others in a VE. As an audience in a VE can induce evaluation apprehension and self-evaluation effects on users, it can influence the perceived stress [19] and workload [6] of users during the accomplishment of a task. In summary, studies demonstrated the impact of a virtual audience on a user depending on its type (i.e., avatar, agent) and the user's perception of it (i.e., evaluative, non-evaluative). Nevertheless, there is a lack of studies that consider the location of the audience, although the issue of physical distance has always been confronted [18]. Today, applications can be shared and users can be located remotely (i.e., distant) or in the same room (i.e., colocated). Therefore, our study proposes to address this topic through analysing how different audience locations affect the impact of social inhibition.

\section{Social inhibition experiment}

The aim of this experiment is to evaluate the impact of audience's location (i.e., colocated, distant) on users in a VE. The audience takes the form of two examiners in the VE to establish the social inhibition of the participant. This paper does not focus on social facilitation because past studies only found results on inhibition [15]. The study is a between-subjects design with one independent variable: the presence of examiners. The participant performs the task according to three conditions: (1) alone (ALONE), (2) with examiners sharing the VE and the same real room than the participant - colocated audience - (IN), (3) with examiners sharing the VE but not the same real room - distant audience - (OUT) (see Fig. 1 ). The evaluation is about performance (objective measures: completion time, and type of answer), stress, cognitive workload and perception of others (subjective measures based on questionnaires). Our hypothesis is that the distant audience has less impact on users than colocated because avatars of the distant audience can be perceived as agents. Co-presence is lower with agents [15], 
reducing evaluation apprehension and self-awareness. Consistent with the experimental design and limitations of previous studies, our hypotheses are:

H1 In the presence of an audience (i.e., IN, OUT), the performance (i.e., types and time of answers) of users will be diminished in comparison to without audience (i.e., ALONE).

H2 In the presence of an audience (i.e., IN, OUT), stress and cognitive workload of users will be higher in comparison to without audience (i.e., ALONE).

H3 Participants will feel the presence of others and their influence more when examiners are physically in a same room (IN) than in a remote room (OUT).

H4 When examiners and users are physically in the same room (IN) than physically in a remote room (OUT) the effects of the audience will be stronger (i.e., poorer performance, higher stress and cognitive workload).
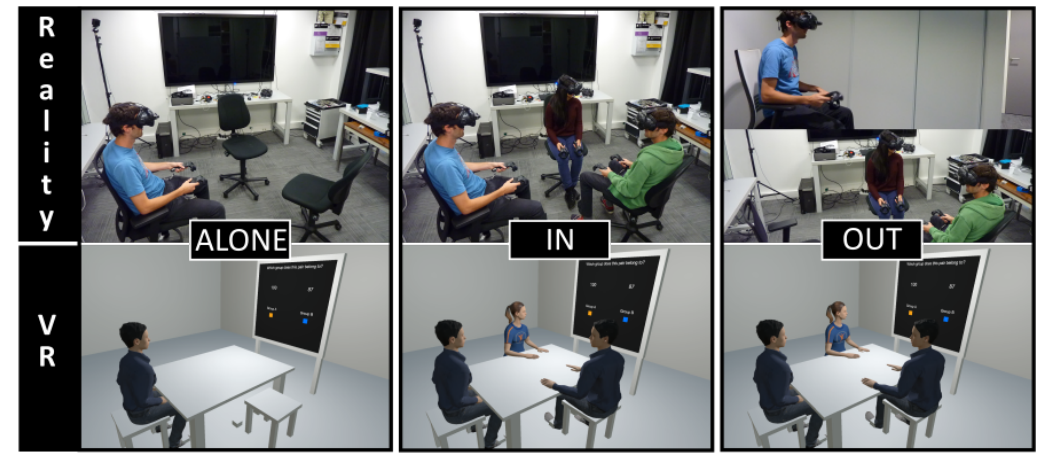

Fig. 1: Setup. User: (left) alone, (middle) audience colocated or (right) distant.

\subsection{Technical Details and Material}

Technical Details. Participants were equipped with an HTC Vive and its two controllers. The VE was a virtual office with a table, a blackboard and chairs (see Fig. 1). Only three people shared the same VE. The application was built in Unity3D with the SteamVR plugin. The VE and interactions were synchronized using a software layer based on Photon Engine 4. Users and examiners were represented by human-like avatars of the same gender (see Fig. 1). We used a T-pose based calibration to adjust the proportion of the avatar for each different user. The avatar animation was based on the rotation and the positions of the two controllers and the HMD using the plugin FinalIK (VRIK) ${ }^{1}$. The skeleton positions were inferred using inverse kinematics.

\footnotetext{
${ }^{1}$ http://root-motion.com
} 
Material. Four questionnaires were used. First, the Short Stress State Questionnaire (SSSQ) [13] measured task engagement, distress and worry using a Likert scale (1-not at all; 5-extremely; 34 questions). Then, the Raw Task Load Index (RTLX) [4] evaluated mental demand, physical demand, temporal demand, effort, performance, and frustration (6 questions). Then, the Slater, Usoh, Steel questionnaire (SUS) [29] measured the feeling of presence using a Likert scale (1-related to low presence; 7 -related to high presence; 6 questions). Finally, the questionnaire regarding the perception of others (QPO) (only for IN and OUT) was based on multiple co-presence questionnaires [26, 24]. It measured two dimensions: perception of the presence of others and their perceived influence (I was in the presence of others; I forget the others and I was focused on the task as if I was alone; I felt observed; My performance was influenced by the presence of others in the VE), and the negative or positive impact of this influence (I was embarrassed by the presence of others in the VE; The presence of others in the VE helped me perform the task; I felt embarrassed by what others might think of me). The QPO uses a Likert scale (1-not at all; 7-extremely; 7 questions).

\subsection{Participants}

The experimentation was carried out with 57 unpaid users: 16 females and 41 males, from 19 to 59 years old $\left(M_{a g e}=35, s d_{a g e}=10\right)$, and with various backgrounds (i.e., students, human resources, engineers, managers, and assistants). Users were split into three groups: 18 participants in the ALONE condition, 20 in the IN condition, and 19 in the OUT condition. 15 users had never experienced VR, 21 users had used VR less than 5 times, 14 users less than 20 times, and 7 users more than 20 times. Demographic characteristics of participants were well distributed among groups.

\subsection{Experimental Design}

The experiment involved two exercises: (1) a short tutorial, (2) the main task (see Fig. 2).

Tutorial. A description of the controller was written on the blackboard: the touchpad was divided in two colored sides, left (orange) and right (blue). Then, users performed a short training during which they were asked to click on the right or left side of the touchpad eight times.

Main Task. It consisted of a categorization of numbers and came from the works of Blascovich et al. [2], and Hoyt et al. [15]. Participants had ten blocks to discover the rules of categorization. Each block included twenty-five trials. For each trial, the participant had 3 seconds to give an answer. Specifically, in each trial, two numbers were displayed on a board in the VE and the user was allowed 3 seconds to say whether the two numbers belonged together in category A or category B. The answer was either correct, categorization found (OK), or incorrect, mistake on the given categorization (NOK), or finally out of time, no answer given before 3 seconds (OT). Audio and visual feedback were given: a soft beep and a green check mark for OK answers or a buzzer and a red-cross for 
NOK and OT answers. Category A numbers followed one normal distribution $(\mu=46.5, \sigma=8$, lower limit $=25$, upper limit $=68)$, and category B numbers followed another one $(\mu=90.7, \sigma=8$, lower limit $=69$, upper limit $=112)$. The numbers were the same for each participant. After each block, the score (as a percentage) was displayed on the blackboard. To complete the task, users needed to obtain eighty percent of correct answers on two consecutive blocks to successfully pass the task. If the user did not find the rule after ten blocks, the task was stopped.

\subsection{Experimental Protocol}

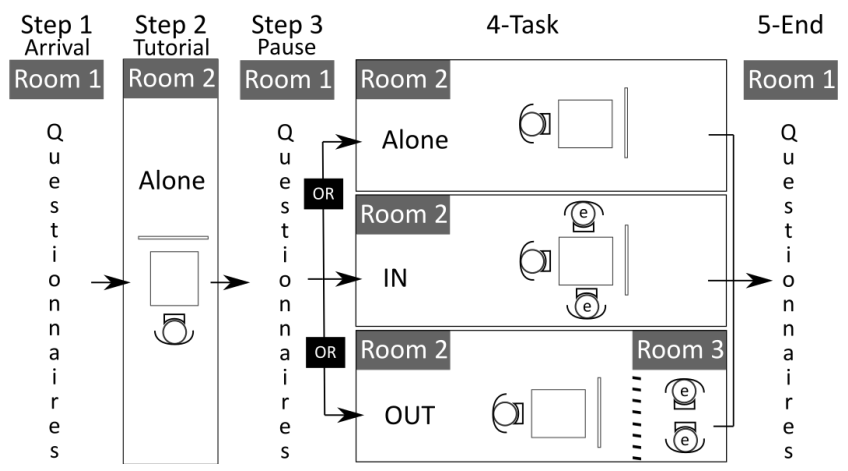

Fig. 2: Design and steps of the experiment

Step 1. All participants read and signed a consent form in which the experiment, its purpose, data recorded, data anonymity, and the possibility to stop the experiment whenever they wanted, were briefly described. Furthermore, each participant filled out a demographic questionnaire.

Step 2. Users were brought into a new room for the tutorial. The instructor gave information for the use of controllers and for the calibration, then equipped the user, left the room and told the user to continue alone. All instructions were given on the blackboard and by a synthesized voice. The instructor came back to notify the user that the tutorial was over.

Step 3. Users were directed to another room to fill out three questionnaires to establish their initial perceived state: the SSSQ, the RTLX, and the SUS. Then participants were redirected to the experiment room.

Step 4. Depending on the condition, participants received different information before performing the main categorization of numbers task. The way to answer was the same as in the tutorial. To elicit more social inhibition, the two examiners (i.e., one female, one male being also the experimenter) were introduced as evaluative. During the task, examiners could only cough or move their arms, hands and head. All noises were caught and played through headphones 
for the three conditions. Examiners were neither allowed to speak nor to answer the user. In the IN condition, participants saw and greeted the examiners before doing the task. In the OUT condition, participants never physically met the examiners. They only knew that they shared the same VE as the examiners, and that avatars embodied real people. Then, examiners equipped themselves and invited the user to continue the experiment. Participants were also informed that their performance could not be observed by any distant person (except the audience) and that the instructor would leave the room in the OUT condition. Instructions and mechanism of the task were displayed on the blackboard before beginning. Quantitative data of performance (dependent variable) were recorded: type of answers - number of correct $(\mathrm{OK})$, incorrect $(\mathrm{NOK})$, and out of time (OT) answers - and answering time (AT; between 0.00s and 3.00s).

Step 5. Participants completed three or four questionnaires (depending on the condition) in order to give their final perceived state: the SSSQ, the RTLX, the SUS, and the QPO.

\subsection{Results}

As all of the trials were performed by each participant (i.e., repeated measures), linear mixed models were used [10]. To evaluate the effect of one variable using linear mixed models, two nested models were compared based on their deviance (chi-square): one without this variable (i.e., the null model), and one with this variable. We compared the effects between the three conditions (i.e., ALONE, IN, and OUT) on levels of stress, cognitive workload, and presence using Kruskal-Wallis (data not normally distributed). Then, we performed an unpaired two-samples t-test (normally distributed data) to analyze differences in the perception levels of others. Only significant results of main or interaction effects $(p<.05)$ are discussed.

Objective Performance To better evaluate variations, differences were measured between the first block performed by users and the last one $(1$ block $=25$ trials, and 1 trial $=3$ s. to categorize 2 displayed numbers). Here are the descriptive results of performance: AT $\left(M_{\text {alone }}=1.29, s d_{\text {alone }}=.71 ; M_{\text {in }}=1.37\right.$, $\left.s d_{\text {in }}=.71 ; M_{\text {out }}=1.54, s d_{\text {out }}=.81\right), \mathrm{OK}\left(M_{\text {alone }}=.70, s d_{\text {alone }}=.46 ;\right.$ $\left.M_{\text {in }}=.68, s d_{\text {in }}=.47 ; M_{\text {out }}=.61, s d_{\text {out }}=.49\right)$, NOK $\left(M_{\text {alone }}=.25\right.$, $\left.s d_{\text {alone }}=.43 ; M_{\text {in }}=.27, s d_{\text {in }}=.44 ; M_{\text {out }}=.30, s d_{\text {out }}=.46\right)$, and $\mathrm{OT}$ $\left(M_{\text {alone }}=.05\right.$, sd $\left.d_{\text {alone }}=.22 ; M_{\text {in }}=.05, s d_{\text {in }}=.22 ; M_{\text {out }}=.08, s d_{\text {out }}=.28\right)$.

Answering Time. The analysis showed an effect of the condition over the AT $\left(\chi^{2}=7.88, p=.019\right)$ and an additive effect of the condition and trial $\left(\chi^{2}=\right.$ $1053.47, p<.001)$. The answering time varied from one trial to an other (see Fig. 3). Comparisons between conditions (post-hoc tests) showed a significant difference between ALONE and OUT $(z=-2.76, p=.015)$, partially supporting H1. There was no significant interaction effect $\left(\chi^{2}=3.23, p=.198\right)$. In other words, the AT was diminished when the audience was distant as opposed to no audience. The comparisons between OUT and IN, and between IN and ALONE 
were not significant; $\mathbf{H 1}$ and $\mathbf{H} \mathbf{4}$ are not supported. The AT was not significantly diminished when the audience was colocated as opposed to no audience, similarly for the distant audience compared to colocated audience.

Type of Answers. We conducted the analyses on the number of OK, NOK, and OT given by participants between models. The value registered was: for OK 0 (i.e., not correct) or 1 (i.e., correct), for NOK 1 (i.e., incorrect) or 0 (i.e., not incorrect), and for OT 1 (i.e., out of time) or 0 (i.e., in time). We used Mixed-Effects Logistic Regression with random effects. The effect of the trial was significant over the number of OK, NOK, OT $\left(\chi_{o k}^{2}=351.45, p_{o k}<.001\right.$; $\left.\chi_{n o k}^{2}=146.81, p_{n o k}<.001 ; \chi_{o t}^{2}=183.49, p_{o t}<.001\right)$. The interaction effects between the condition and the trial (see Fig. 3) were also significant $\left(\chi_{o k}^{2}=\right.$ $\left.15.25, p_{o k}<.001 ; \chi_{n o k}^{2}=11.09, p_{n o k}=.004 ; \chi_{o t}^{2}=18.33, p_{o t}<.001\right)$. The number of OK grew quicker in the ALONE condition, than in the IN and also in the OUT. The number of NOK and OT decreased quicker in the ALONE condition, then in the IN and finally in the OUT. This interaction between conditions and trials (CONDI*TRIAL) in these three variables support $\mathbf{H 1}$, but not H4. In other words, the evolution of the type of answers over trials was significantly different in relation to the location of the audience. Specifically, the number of OK increased more slowly over the trials in the presence of the audience (i.e., IN or OUT) as opposed to without audience (i.e., ALONE) but the evolution was quicker in the presence of the colocated audience compared to the distant audience. Furthermore, the number of NOK and OT decreased more slowly over trials in the presence of the audience (i.e., IN or OUT) as opposed to without the audience (i.e., ALONE) but the evolution was quicker in the presence of the colocated audience compared to the distant audience.
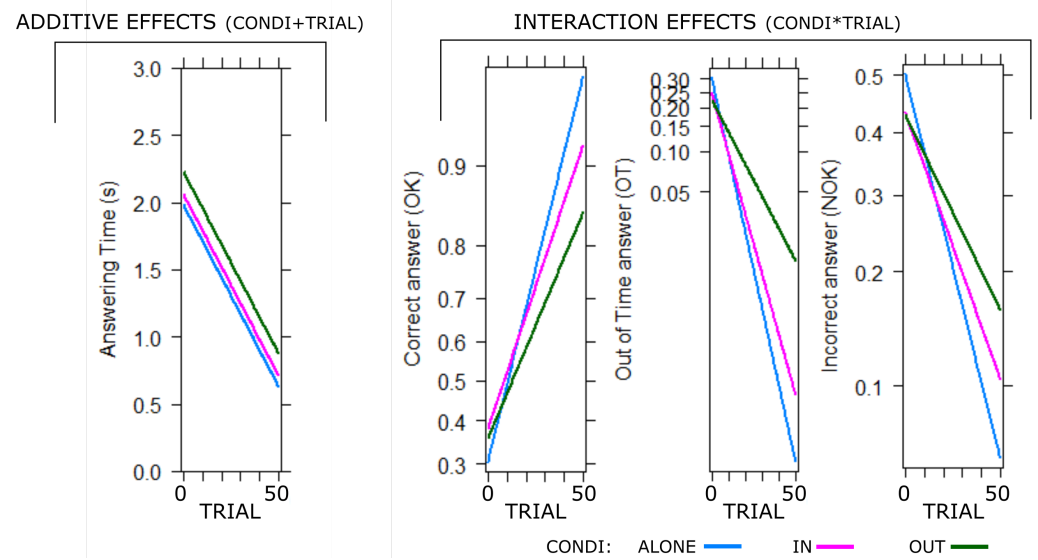

Fig. 3: Additive and interaction effects of Trial and Condition on answering time and on type of answers 
Subjective Feelings and Perceptions Perception of Others. Using the QPO, the t-test showed a main effect on the Perception of Others and their Influence $(t(31.33)=2.43, p=.021)$. Users perceived the examiners more significantly and felt more influenced by them when they were physically colocated $\left(M_{i n}=4.99\right.$, $\left.s d_{\text {in }}=0.83\right)$ than distant $\left(M_{\text {out }}=4.09, s d_{\text {out }}=1.40\right)$. No other significant difference was found. H3 is supported by these results.

Stress. Kruskal-Wallis tests did not show significant differences regarding all dimensions of the SSSQ: Engagement $\left(M_{\text {alone }}=3.75\right.$, sd $d_{\text {alone }}=1.13 ; M_{\text {in }}=$ $\left.3.46, s d_{\text {in }}=1.11 ; M_{\text {out }}=3.54, s d_{\text {out }}=1.30\right)$, Worry $\left(M_{\text {alone }}=1.78, s d_{\text {alone }}=\right.$ $\left.1.02 ; M_{\text {in }}=1.99, s d_{\text {in }}=1.17 ; M_{\text {out }}=2.12, s d_{\text {out }}=1.22\right)$, and Distress $\left(M_{\text {alone }}=1.83, s d_{\text {alone }}=1.07 ; M_{\text {in }}=1.69, s d_{\text {in }}=1.03 ; M_{\text {out }}=1.86\right.$, $\left.s d_{\text {out }}=1.10\right)$. $\mathbf{H} 2$ and $\mathbf{H} 4$ are not supported by these results. In other words, participants' stress levels were not significantly affected by audience location.

Cognitive workload. The Kruskal-Wallis tests did not find significant differences regarding all dimensions of the RTLX $(M \epsilon[15.00 ; 62.00])$. H2 and $\mathbf{H 4}$ are not supported by these results. In other words, participants' workload was not significantly affected by audience location.

Presence. The one-way ANOVA test did not find significant differences: $M_{\text {alone }}=3.5, s d_{\text {alone }}=2.05, M_{\text {in }}=4.10, s d_{\text {in }}=1.88, M_{\text {out }}=4.17, s d_{\text {out }}=$ 1.79 .

\section{Discussion}

The results show that the presence of examiners influenced the participant's natural performance improvement (i.e., answering time, and type of answers) when doing a repeated task. Firstly, objective measures show a significant improvement of correct answers within the time frame as well as a reduction of false and out of time answers. This can be explained by a natural improvement due to repetitions during the task. But the improvement is not the same between conditions. The interaction effects between the audience and the trials showed that the evolution within the time frame, positive or negative (depending of the objective measure) was slower when an audience was present compared to absent. The manifestation of social inhibition among users due to the presence of an audience seemed to result in a decrease of the natural performance evolution during this specific task. Our results are consistent with previous social inhibition studies [15]. Thus, H1 is partially supported because results on answering time show no clear distinction between with or without audience. The only significant difference is between the distant and the colocated audience.

Secondly, social inhibition is not perceivable in cognitive workload and stress. The results of questionnaires did not demonstrate any differences despite the anxiety of the evaluation that could be induced by the examiners. Thus, $\mathbf{H 2}$ is not supported. This result could be due to the variability of mathematical skill of participants [17]. Indeed, perception and resources used to perform the task may vary between participants. Unfortunately, mathematical aptitude was not evaluated in the current study. 
Thirdly, results comparing the subjective perception of others between colocated (i.e., IN) and distant (i.e., OUT) audiences show significant differences. Participants seemed to perceive the examiners more significantly and seemed to feel more influenced by them when they were physically colocated than when they were distant. Thus, H3 is supported. However, participants did not find significant differences between conditions that resulted in a negative influence. Moreover, the overall score of the negative influence of examiners was not high probably because examiners were passive during the experiment and did not cause any additional stress.

Finally, users' performance refutes $\mathbf{H} 4$. Statistical analysis present significant differences between conditions (i.e., IN, and OUT). Indeed, previous interaction effects (i.e., audience over trial) also show that the positive or negative evolution of the performance was even slower when users and audience were distant. Therefore, a stronger social inhibition seems to occur in distant users compared to users who were next to one another. This is most likely the explanation for the slower natural performance improvement (i.e., type of answers) of participants. The results seem to indicate that the reason users negatively experience their performance (when everyone is in the same real room) is not related to the extent to which they perceive or feel the presence of others. To sum up, the performance of participants seems to be affected by the presence of an audience and the type of the audience (i.e., IN, OUT). Furthermore, the significance of the perception of others seems to also depend on the type of the audience.

\section{Conclusion}

In this paper, we explored the effects of social inhibition induced by the presence of examiners on a user in a VE. In VR, the user had to perform a unknown and challenging task.

Analyses focused on performance, stress, users' cognitive workload and their perception of others. Three types of audience were evaluated: no-audience, physically colocated examiners embodied by avatars in the application, and physically distant examiners also embodied by avatars.

Firstly, the results show a difference between users' reactions when they are in the presence of others compared to no-audience in the VE. Indeed, the natural performance improvement occurred more slowly when the audience was colocated than with no audience, and even more slowly with a distant audience than a colocated audience. A slower improvement with a distant audience could be due to a weaker perception of others compared to a colocated audience.

More generally, users in VR seem to be affected by social inhibition in the presence of an audience, and they seem to be even more affected when the audience is physically distant, in a remote room. In this way a learning or training process using repetition could be influenced by an audience. Designers of such applications should pay attention to the setup before building the application. It could be interesting to explore the variation of the social inhibition through the same audience types but with a different task (e.g., fire drills, training for 
maintenance, evaluation of a service) or with an audience not present in the VE but physically present in the same room as the participant (e.g., a demonstration in an exhibition).

\section{References}

1. Blascovich, J., Loomis, J., Beall, A.C., Swinth, K.R., Hoyt, C.L., Bailenson, J.N.: Target article: Immersive virtual environment technology as a methodological tool for social psychology. Psychological Inquiry 13(2), 103-124 (2002)

2. Blascovich, J., Mendes, W.B., Hunter, S.B., Salomon, K.: Social "facilitation" as challenge and threat. Journal of personality and social psychology 77(1), 68 (1999)

3. Bond, C.F., Titus, L.J.: Social facilitation: A meta-analysis of 241 studies. Psychological bulletin 94(2), 265 (1983)

4. Byers, J.C.: Traditional and raw task load index (tlx) correlations: Are paired comparisons necessary? Advances in Industrial Ergonomics and Safety l: Taylor and Francis (1989)

5. Chartrand, T.L., Bargh, J.A.: The chameleon effect: the perception-behavior link and social interaction. Journal of personality and social psychology $\mathbf{7 6}(6), 893$ (1999)

6. Claypoole, V.L., Dewar, A.R., Fraulini, N.W., Szalma, J.L.: Effects of social facilitation on perceived workload, subjective stress, and vigilance-related anxiety. Proceedings of the Human Factors and Ergonomics Society Annual Meeting 60(1), 1169-1173 (2016)

7. Cottrell, N.B., Wack, D.L., Sekerak, G.J., Rittle, R.H.: Social facilitation of dominant responses by the presence of an audience and the mere presence of others. Journal of personality and social psychology 9(3), 245 (1968)

8. Dijksterhuis, A., Bargh, J.A.: The perception-behavior expressway: Automatic effects of social perception on social behavior. In: Advances in Experimental Social Psychology, vol. 33, pp. 1 - 40. Academic Press (2001)

9. Emmerich, K., Masuch, M.: Watch me play: Does social facilitation apply to digital games? In: Proceedings of the 2018 CHI Conference on Human Factors in Computing Systems. pp. 100:1-100:12. CHI '18 (2018)

10. Gueorguieva, R., Krystal, J.H.: Move over anova: Progress in analyzing repeatedmeasures data and its reflection in papers published in the archives of general psychiatry. Archives of General Psychiatry 61(3), 310-317 (Mar 2004)

11. Guerin, B., Innes, J.M.: Social facilitation and social monitoring: A new look at zajonc's mere presence hypothesis. British Journal of Social Psychology 21(1), 7-18 (1982)

12. Guerin, B.: Mere presence effects in humans: A review. Journal of experimental social psychology 22(1), 38-77 (1986)

13. Helton, W.S., Näswall, K.: Short stress state questionnaire. European Journal of Psychological Assessment 31(1), 20-30 (jun 2015)

14. Henchy, T., Glass, D.C.: Evaluation apprehension and the social facilitation of dominant and subordinate responses. Journal of personality and social psychology 10(4), 446 (1968)

15. Hoyt, C.L., Blascovich, J., Swinth, K.R.: Social inhibition in immersive virtual environments. Presence: Teleoperators and Virtual Environments 12(2), 183-195 (2003) 
16. Innes, J.M., Young, R.F.: The effect of presence of an audience, evaluation apprehension and objective self-awareness on learning. Journal of Experimental Social Psychology (1975)

17. Jamieson, J.P., Peters, B.J., Greenwood, E.J., Altose, A.J.: Reappraising stress arousal improves performance and reduces evaluation anxiety in classroom exam situations. Social Psychological and Personality Science 7(6), 579-587 (2016)

18. Knowles, E.S.: Social physics and the effects of others: Tests of the effects of audience size and distance on social judgments and behavior. Journal of Personality and Social Psychology 45(6), 1263 (1983)

19. Kushnir, T.: Stress and social facilitation: the effects of the presence of an instructor on student nurses behaviour. Journal of Advanced Nursing 11(1), 13-19 (1986)

20. Lemoine, J.E., Roland-Lvy, C.: The effect of the presence of an audience on risktaking while gambling: the social shield. Social Influence 12(2-3), 101-114 (2017)

21. Sanchez-Vives, M.V., Slater, M.: From presence to consciousness through virtual reality. Nature Reviews Neuroscience 6(4), 332 (2005)

22. Schroeder, R.: Being there together and the future of connected presence. Presence: Teleoperators and Virtual Environments 15(4), 438-454 (2006)

23. Skarratt, P.A., Cole, G.G., Kingstone, A.: Social inhibition of return. Acta Psychologica 134(1), 48 - 54 (2010)

24. Slater, M., Sadagic, A., Usoh, M., Schroeder, R.: Small-group behavior in a virtual and real environment: A comparative study. Presence: Teleoperators and Virtual Environments 9(1), 37-51 (2000)

25. Smeesters, D., Wheeler, S.C., Kay, A.C.: Indirect prime-to-behavior effects: The role of perceptions of the self, others, and situations in connecting primed constructs to social behavior. In: Advances in Experimental Social Psychology, vol. 42, pp. 259 - 317. Academic Press (2010)

26. Steed, A., Slater, M., Sadagic, A., Bullock, A., Tromp, J.: Leadership and collaboration in shared virtual environments. In: Proceedings IEEE Virtual Reality (Cat. No. 99CB36316). pp. 112-115 (March 1999)

27. Stotland, E., Zander, A.: Effects of public and private failure on self-evaluation. The Journal of Abnormal and Social Psychology 56(2), 223-229 (1958)

28. Swinth, K.R., Blascovich, J.: Perceiving and responding to others: Human-human and human-computer social interaction in collaborative virtual environments. In: Proceedings of the 5th Annual International Workshop on PRESENCE. vol. 392 (2002)

29. Usoh, M., Catena, E., Arman, S., Slater, M.: Using presence questionnaires in reality. Presence: Teleoperators \& Virtual Environments 9(5), 497-503 (2000)

30. Velho, L., Lucio, D., Carvalho, L.: Situated participatory virtual reality. In: In Proceedings of XVI Simposio Brasileiro de Jogos e Entretenimento Digital (2017)

31. Wienrich, C., Gross, R., Kretschmer, F., Mller-Plath, G.: Developing and proving a framework for reaction time experiments in vr to objectively measure social interaction with virtual agents. In: 2018 IEEE Conference on Virtual Reality and 3D User Interfaces (VR). pp. 191-198 (March 2018)

32. Wolf, L.K., Bazargani, N., Kilford, E.J., Dumontheil, I., Blakemore, S.J.: The audience effect in adolescence depends on who's looking over your shoulder. Journal of Adolescence 43, 5- 14 (2015)

33. feng $\mathrm{Yu}, \mathrm{R} ., \mathrm{Wu}, \mathrm{X}$. : Working alone or in the presence of others: exploring social facilitation in baggage x-ray security screening tasks. Ergonomics 58(6), 857-865 (2015)

34. Zajonc, R.B.: Social facilitation. Science 149(3681), 269-274 (1965) 